\title{
The Faddeev Equation and the Essential Spectrum of a Model Operator Associated with the Hamiltonian of a Nonconserved Number of Particles
}

\author{
Zahriddin Muminov, ${ }^{1}$ Fudziah Ismail, ${ }^{1,2}$ and Jamshid Rasulov ${ }^{3}$ \\ ${ }^{1}$ Department of Mathematics, Faculty of Science, University Putra Malaysia, 43400 Serdang, Malaysia \\ ${ }^{2}$ Institute for Mathematical Research, University Putra Malaysia, 43400 Serdang, Malaysia \\ ${ }^{3}$ Academic Lyceum Number One Under Samarkand Institute of Economics and Service, 140104 Samarkand, Uzbekistan \\ Correspondence should be addressed to Zahriddin Muminov; zimuminov@gmail.com
}

Received 13 February 2014; Accepted 9 April 2014; Published 2 June 2014

Academic Editor: Pavel Kurasov

Copyright (c) 2014 Zahriddin Muminov et al. This is an open access article distributed under the Creative Commons Attribution License, which permits unrestricted use, distribution, and reproduction in any medium, provided the original work is properly cited.

\begin{abstract}
A model describing a truncated operator $H$ (truncated with respect to the number of particles) and acting in the direct sum of zero-, one-, and two-particle subspaces of fermionic Fock space $\mathscr{F}_{a}\left(L^{2}\left(\mathbb{T}^{3}\right)\right)$ over $L^{2}\left(\mathbb{T}^{3}\right)$ is investigated. The location of the essential spectrum of the model operator $H$ is described by means of the spectrum of the Friedreich model $h(p) ; p \in \mathbb{T}^{3}$. Moreover, for the resolvent of $H$, the Faddeev type system of integral equations is obtained.
\end{abstract}

\section{Introduction}

The main goal of the present paper is to give a thorough mathematical treatment of some properties for a model operator $H$ with emphasis on the essential spectrum and its resolvent. This operator, associated to a system describing two identical fermions and one particle of another nature in interactions, without conservation of the number of particles on the three-dimensional lattice, acts in the direct sum of zero-, one-, and two-particle subspaces of fermionic Fock space $\mathscr{F}_{a}\left(L^{2}\left(\mathbb{T}^{3}\right)\right)$ over $L^{2}\left(\mathbb{T}^{3}\right)$.

In statistical physics Minlos and Spohn [1], Malyshev and Minlos [2], solid-state physics Mattis [3], Mogilner [4] and the theory of quantum fields Friedrichs [5], Buhler et al. [6] some important problems arise where the number of quasiparticles is bounded, but not fixed. Sigal et al. [7] have developed geometric and commutator techniques to find the location of the spectrum and to prove absence of singular continuous spectrum for Hamiltonians without conservation of the particle number.

Notice that the study of systems describing $n$ particles in interaction, without conservation of the number of particles, is reduced to the investigation of the spectral properties of self-adjoint operators acting in the cut subspace $\mathscr{H}^{(n)}$ of the Fock space, consisting of $r \leq n$ particles $[1,4-8]$.

In the works [9-16], the location of essential spectra of model operators, associated with a system describing three particles, without conservation of the number of particles, was investigated. However, the corresponding operators act in the direct sum of zero-, one-, and two-particle subspaces of Fock space or bosonic Fock space, in some cases (see, e.g., $[9,10])$ over $L^{2}\left(\mathbb{T}^{3}\right)$. We also refer to [17] for essential spectrum of discrete Schrödinger operators, associated to a system of two identical fermions and one particle of another nature in interactions.

Recently, the authors in $[18,19]$ have proved that the fermionic Fock space case has some assertions being related to the Efimov effect (infinite number of bound states if the associated generalized Friedrichs model (FM) has a threshold resonance). These results show that this effect does not hold even this FM has a threshold resonance while it holds in the (bosonic) Fock space case. This fact pushes us to study the resolvent and essential spectrum of the investigating operator.

In the present paper, under some smoothness assumptions we obtain the location of the essential spectrum of the model operator $H$, described by means of the spectrum 
of the Friedreich model $h(p), p \in \mathbb{T}^{3}$, and we derive the Faddeev type system of integral equations for the components of the resolvent of this model operator and find the form for resolvent.

The organization of the present paper is as follows. Section 1 is an introduction to the whole work. In Section 2, the model operator is described as a bounded self-adjoint operator $H$ in $\mathscr{H}^{(3)}$. Some spectral properties of the corresponding channel operator and Friedrichs models $H(p)$, $p \in \mathbb{T}^{3}$, are given in Section 3. Section 4 deals with the review of the Faddeev type system of integral equations for the eigenfunction of operator $H$. In Section 5, we represent the main results (Theorems 9 and 11) and the sketch of their proofs.

\section{Conventions and Definition of the Model Operator}

Let $\mathbb{T}^{3}$ be the three-dimensional torus, the cube $(-\pi, \pi]^{3}$ with appropriately identified sides. We remark that the torus $\mathbb{T}^{3}$ will always be considered as an abelian group with respect to the addition and multiplication by the real numbers regarded as operations on $\mathbb{R}^{3}$ modulo $(2 \pi \mathbb{Z})^{3}$. Denote by $L_{\text {as }}^{2}\left(\left(\mathbb{T}^{3}\right)^{2}\right)$ the subspace of antisymmetric functions of the Hilbert space $L^{2}\left(\left(\mathbb{T}^{3}\right)^{2}\right)$.

We also introduce the following notations:

$$
\mathscr{H}_{0}=\mathbb{C}, \quad \mathscr{H}_{1}=L^{2}\left(\mathbb{T}^{3}\right), \quad \mathscr{H}_{2}=L_{\text {as }}^{2}\left(\left(\mathbb{T}^{3}\right)^{2}\right),
$$

and let $I_{j}$, respectively, $\langle\cdot, \cdot\rangle_{j}$ be an identity operator, respectively, an inner product in $\mathscr{H}_{j}, j=0,1,2$.

The Hilbert space $\mathscr{H}^{(3)}=\mathscr{H}_{0} \oplus \mathscr{H}_{1} \oplus \mathscr{H}_{2}$ is called the direct sum of zero-, one-, and two-particle subspaces of fermionic Fock space $\mathscr{F}_{a}\left(L^{2}\left(\mathbb{T}^{3}\right)\right)$ over $L^{2}\left(\mathbb{T}^{3}\right)$.

For $g \in L^{2}\left(\mathbb{T}^{3}\right)$ we define the following operator:

$$
\begin{aligned}
& \mathbf{g}: L^{2}\left(\mathbb{T}^{3}\right) \longrightarrow \mathbb{C}, \quad \mathbf{g}\left(f_{1}\right)=\left\langle f_{1}, g\right\rangle_{1}, \quad f_{1} \in L^{2}\left(\mathbb{T}^{3}\right), \\
& \mathbf{g}^{*}: \mathbb{C} \longrightarrow L^{2}\left(\mathbb{T}^{3}\right), \quad \mathbf{g}^{*} f_{0}=g(\cdot) f_{0}, \quad f_{0} \in \mathbb{C}, \\
& L_{g}, L_{s g}: L^{2}\left(\left(\mathbb{T}^{3}\right)^{2}\right) \longrightarrow L^{2}\left(\mathbb{T}^{3}\right), \\
& L_{g}=I_{1} \otimes \mathbf{g}, \quad L_{s g}=\mathbf{g} \otimes I_{1}, \\
& L_{g}^{*}, L_{s g}^{*}: L^{2}\left(\mathbb{T}^{3}\right) \longrightarrow L^{2}\left(\left(\mathbb{T}^{3}\right)^{2}\right), \\
& L_{g}^{*}=I_{1} \otimes \mathbf{g}^{*}, \quad L_{s g}^{*}=\mathbf{g}^{*} \otimes I_{1} .
\end{aligned}
$$

Let $H_{i j}$ be annihilation (creation) operators [5] defined in the Fock space for $i<j(i>j)$. We note that, in physics, an annihilation operator is an operator that lowers the number of particles in a given state by one; a creation operator being an adjoint of the annihilation operator is an operator that increases the number of particles in a given state by one.

In this paper, we consider the case, where the number of annihilations and creations of the particles of the considering system is equal to 1 . It means that $H_{i j} \equiv 0$ for all $|i-j|>1$. So, a model describing a truncated operator $H$ acts in the Hilbert space $\mathscr{H}^{(3)}$ as a matrix operator and given by

$$
H=\left(\begin{array}{ccc}
H_{00} & H_{01} & 0 \\
H_{10} & H_{11} & H_{12} \\
0 & H_{21} & H_{22}
\end{array}\right),
$$

where

$$
\begin{aligned}
& H_{01}=\mathbf{a}, \quad H_{10}=\mathbf{a}^{*}, \\
& H_{12}=L_{b}, \quad H_{11}=H_{11}^{0}, \\
& H_{21}=\frac{1}{2}\left(L_{b}^{*}-L_{s b}^{*}\right), \\
& H_{22}=H_{22}^{0}-L_{\varphi}^{*} L_{\varphi}-L_{s \varphi}^{*} L_{s \varphi},
\end{aligned}
$$

the operators $H_{00}, H_{11}^{0}$, and $H_{22}^{0}$ are multiplication by the functions $u_{0}, u(\cdot)$, and $E(\cdot, \cdot)$ in $\mathscr{H}_{0}, \mathscr{H}_{1}$, and $\mathscr{H}_{2}$, respectively.

Here $u_{0}$ is a fixed number; $a(\cdot), b(\cdot), u(\cdot), \varphi(\cdot)$ are realvalued continuous functions on $\mathbb{T}^{3} ; E(\cdot, \cdot)$ is a real-valued continuous symmetric function on $\left(\mathbb{T}^{3}\right)^{2}$.

Remark that under these conditions the operator $H$ is bounded and self-adjoint.

\section{The Spectrum of the Channel Operator and of the Friedrichs Model}

To study the essential spectrum, along with the operator $H$, we also consider a bounded self-adjoint operator $H_{\mathrm{ch}}$ acting in $\widehat{\mathscr{H}}=L^{2}\left(\mathbb{T}^{3}\right) \oplus L^{2}\left(\left(\mathbb{T}^{3}\right)^{2}\right)$ with form

$$
H_{\mathrm{ch}}=\left(\begin{array}{cc}
H_{11} & \frac{1}{\sqrt{2}} L_{b} \\
\frac{1}{\sqrt{2}} L_{b}^{*} & H_{22}^{0}-L_{\varphi}^{*} L_{\varphi}
\end{array}\right) .
$$

This operator has a characteristic property of a channel operator (see, e.g., [20]) of three-particle discrete Schrödinger operator. Therefore, we call it a channel operator, corresponding to the model operator $H$. Note that the channel operator $H_{\text {ch }}$ has a simpler structure than the $H$, and therefore the study of the spectral properties of $H_{\text {ch }}$ plays an important role in future studies of the spectrum of $H$.

Since $H_{\text {ch }}$ commutes with the abelian group of multiplication operators $U_{\alpha}$, by the function $\alpha(\cdot)$ :

$$
U_{\alpha}\left(\begin{array}{c}
f_{1}(p) \\
f_{2}(p, q)
\end{array}\right)=\left(\begin{array}{c}
\alpha(p) f_{1}(p) \\
\alpha(p) f_{2}(p, q)
\end{array}\right), \quad \alpha \in L^{2}\left(\mathbb{T}^{3}\right),
$$

the decomposition of $\widehat{\mathscr{H}}$ into $\widehat{\mathscr{H}}=\int_{p \in \mathbb{T}^{3}} \mathscr{H}^{(2)} d p$, where $\mathscr{H}^{(2)}=\mathscr{H}_{0} \oplus \mathscr{H}_{1}$, implies that the operator $H_{\text {ch }}$ is decomposed into a direct von Neumann integral (see, e.g., [21, Theorem XIII.84]):

$$
H_{\mathrm{ch}}=\int_{\mathbb{T}^{3}} \oplus H(p) d p
$$

Here $H(p), p \in \mathbb{T}^{3}$, is a Friedrichs model being bounded, self-adjoint, and acting in $\mathscr{H}^{(2)}$ by the rule

$$
H(p)=H_{0}(p)+V,
$$


where

$$
H_{0}(p)=\left(\begin{array}{cc}
0 & 0 \\
0 & h_{0}(p)
\end{array}\right), \quad V=\left(\begin{array}{cc}
u(p) & \frac{1}{\sqrt{2}} \mathbf{b} \\
\frac{1}{\sqrt{2}} \mathbf{b}^{*} & -\boldsymbol{\varphi}^{*} \boldsymbol{\varphi}
\end{array}\right),
$$

and $h_{0}(p), p \in \mathbb{T}^{3}$, is a multiplication operator by the function $e_{p}(\cdot):=E(p, \cdot):$

$$
\left(h_{0}(p) f_{1}\right)(q)=e_{p}(q) f_{1}(q), \quad f_{1} \in \mathscr{H}_{1} .
$$

Remark 1. The spectral properties of such type of Friedrichs models are studied in $[9,10]$.

According to the theorem on the spectrum of decomposable operators (see, e.g., [21, Theorem XIII.85]) from (7), we obtain the following.

Theorem 2. For the essential spectrum $\sigma_{\text {ess }}(H)$ of the operator $H$ the equality,

$$
\sigma\left(H_{c h}\right)=\cup_{p \in \mathbb{T}^{3}}\left\{\sigma_{d}(H(p))\right\} \cup\left[E_{\min }, E_{\max }\right],
$$

holds, where $\sigma_{d}(H(p))$ is the discrete spectrum of the operator $H(p), p \in \mathbb{T}^{3}$, and

$$
E_{\min }=\min _{p, q \in \mathbb{T}^{3}} E(p, q), \quad E_{\max }=\max _{p, q \in \mathbb{T}^{3}} E(p, q) .
$$

3.1. The Spectral Properties of the Friedrichs Model H(p), $p \in$ $\mathbb{T}^{3}$. Since rank $V \leq 3$, and then in accordance with the stability of the essential spectrum under finite rank perturbations, the essential spectrum $\sigma_{\text {ess }}(h(p))$ of $h(p)$ coincides with the spectrum of $h_{0}(p)$, and, namely, the equality,

$$
\sigma_{\text {ess }}(H(p))=\sigma_{\text {ess }}\left(h_{0}(p)\right)=[m(p), M(p)], \quad p \in \mathbb{T}^{3},
$$

holds, where $m(p)$ and $M(p)$ are defined by

$$
m(p)=\min _{q \in \mathbb{T}^{3}} e_{p}(q), \quad M(p)=\max _{q \in \mathbb{T}^{3}} e_{p}(q) .
$$

For any $p \in \mathbb{T}^{3}$ we define an analytic function $\Delta(p, z)$ (the Fredholm determinant associated with the operator $H(p))$ in $\mathbb{C} \backslash[m(p), M(p)]$ by

$$
\begin{aligned}
\Delta(p ; z)= & \left(I_{0}-\varphi r_{0}(p, z) \varphi^{*}\right) \\
& \times\left(H_{00}(p)-z I_{0}-\frac{1}{2} \mathbf{b} r_{0}(p, z) \mathbf{b}^{*}\right) f_{0} \\
& -\frac{1}{2}\left(\mathbf{b} r_{0}(p, z) \varphi^{*}\right)^{2} f_{0}, \quad f_{0} \equiv 1 ;
\end{aligned}
$$

that is,

$$
\begin{aligned}
\Delta(p ; z)= & \left(1-\int_{\mathbb{T}^{3}} \frac{\varphi^{2}(s) d s}{E(p, s)-z}\right) \\
& \times\left(u(p)-z-\frac{1}{2} \int_{\mathbb{T}^{3}} \frac{b^{2}(s) d s}{E(p, s)-z}\right) \\
& -\frac{1}{2}\left(\int_{\mathbb{T}^{3}} \frac{b(s) \varphi(s) d s}{E(p, s)-z}\right)^{2},
\end{aligned}
$$

where $r_{0}(p ; z), z \in \mathbb{C} \backslash[m(p), M(p)]$, is the resolvent of $h_{0}(p), p \in \mathbb{T}^{3}$.

Lemma 3. For any $p \in \mathbb{T}^{3}$ the number $z \in \mathbb{C} \backslash[m(p), M(p)]$ is an eigenvalue of $H(p)$ if and only if $\Delta(p, z)=0$.

Proof. The equation

$$
H(p) f=z f, \quad f \in \mathscr{H}^{(2)},
$$

that is, the system of equations,

$$
\begin{aligned}
&(u(p)-z) f_{0}+\frac{1}{\sqrt{2}} \mathbf{b} f_{1}=0, \\
&-\frac{1}{\sqrt{2}} r_{0}(p ; z) \mathbf{b}^{*} f_{0}+r_{0}(p ; z) \boldsymbol{\varphi}^{*} \boldsymbol{\varphi} f_{1}=f_{1}, \\
& \quad f=\left(f_{0}, f_{1}\right) \in \mathscr{H}^{(2)},
\end{aligned}
$$

is equivalent to the system,

$$
\begin{gathered}
\left(H_{00}(p)-z I_{0}-\frac{1}{2} \mathbf{b} r_{0}(p, z) \mathbf{b}^{*}\right) f_{0} \\
+\frac{1}{\sqrt{2}} \mathbf{b} r_{0}(p, z) \boldsymbol{\varphi}^{*} \alpha=0 \\
-\frac{1}{\sqrt{2}} \boldsymbol{\varphi} r_{0}(p, z) \mathbf{b}^{*} f_{0} \\
+\left(I_{0}-\boldsymbol{\varphi} r_{0}(p, z) \boldsymbol{\varphi}^{*}\right) \alpha=0, \\
f_{0}, \alpha \in \mathbb{C} .
\end{gathered}
$$

The solutions of (17) and (19) are connected with relations

$$
\begin{gathered}
f_{0}=f_{0}, \quad \alpha=\varphi f_{1}, \\
f_{1}(q)=r_{0}(p ; z)\left(-\frac{1}{2} b(q) f_{0}+\varphi(q) \alpha\right) .
\end{gathered}
$$

Since the determinant of (19) is equal to $\Delta(p ; z)$, the equation, $H(p) f=z f, f \in \mathscr{H}^{(2)}$, has nontrivial solution if and only if $\Delta(p ; z)=0$.

Denote by $n_{-}(H(p), z), z \leq \inf \sigma_{\text {ess }}(H(p))$, (resp., $\left.n_{+}(H(p), z), z \geq \sup \sigma_{\text {ess }}(H(p))\right)$ the number of eigenvalues, counted according their multiplicities of $H(p)$ lying below $z$ (resp., above $z$ ).

Lemma 4. For any fixed $p \in \mathbb{T}^{3}$, the following are true:

(a) if $\varphi(\cdot)$ and $b(\cdot)$ are linearly dependent (independent), then

$$
n_{-}(H(p), m(p)) \leq 1 \quad\left(n_{-}(H(p), m(p)) \leq 2\right) ;
$$

(b)

$$
n_{+}(H(p), M(p)) \leq 1 \text {. }
$$


Proof. Simple calculation gives that the numbers

$$
z_{1,2}=-\frac{u(p) \pm \sqrt{u^{2}(p)+4\|b\|^{2}}}{2} \quad z_{1}<0<z_{2}
$$

are simple eigenvalues of

$$
V^{\prime}=\left(\begin{array}{cc}
u(p) & \frac{1}{\sqrt{2}} \mathbf{b} \\
\frac{1}{\sqrt{2}} \mathbf{b}^{*} & 0
\end{array}\right)
$$

Since Range $(V)=\mathbb{C} \oplus\langle b, \varphi\rangle$, where $\langle b, \varphi\rangle$ is the subspace spanned by $b$ and $\varphi$, we have $\operatorname{dimRange}(V)=3$ if $\varphi(\cdot)$ and $b(\cdot)$ are linearly independent and dimRange $(V)=2$ if $\varphi(\cdot)$ and $b(\cdot)$ are linearly dependent.

Then, using $-\boldsymbol{\varphi}^{*} \boldsymbol{\varphi} \leq 0, \sigma_{\mathrm{ess}}\left(V^{\prime}\right)=\{0\}$ for the operator $V$, we obtain the following:

(i) if $\varphi(\cdot)$ and $b(\cdot)$ are linearly independent, then $V$ has two negative eigenvalues (with multiplicities) and a unique (simple) positive one;

(ii) if $\varphi(\cdot)$ and $b(\cdot)$ are linearly independent, then $V$ has only one (simple) negative and one (simple) positive eigenvalue.

According to $H(p) \geq m(p)+V, H(p) \leq M(p)+V$, and the minimax principle the number $n_{-}(H(p), m(p))$ (resp., $\left.n_{+}(H(p), M(p))\right)$ satisfies

$$
\begin{aligned}
& n_{-}(H(p), m(p)) \leq n_{-}(m(p)+V, m(p)) \\
& \left(\text { resp., } n_{+}(H(p), M(p)) \leq n_{+}(M(p)+V, M(p))\right) .
\end{aligned}
$$

Hence, using (i), (ii), and $n_{-}(m(p)+V, m(p))=n_{-}(V, 0)$, $n_{+}(M(p)+V, M(p))=n_{+}(V, 0)$ we get proof.

Corollary 5. Let $p \in \mathbb{T}^{3}$. Consider the following:

(a) if $\varphi(\cdot)$ and $b(\cdot)$ are linearly (dependent) independent, then the function $\Delta(p, \cdot)$ can have no more than one zero (two zeros) in the interval $(-\infty, m(p))$;

(b) $\Delta(p, \cdot)$ can have only one zero in the interval $(M(p), \infty)$.

\section{The Faddeev Type Equation}

Set

$$
\Sigma=\left[E_{\min }, E_{\max }\right] \cup \sigma_{\text {two }},
$$

where

$$
\begin{aligned}
\sigma_{\mathrm{two}}=\{z: z \in \mathbb{R} \backslash[m(p), M(p)] \\
\left.\Delta(p ; z)=0 \text { for some } p \in \mathbb{T}^{3}\right\} .
\end{aligned}
$$

Clearly, according to Theorem 2 and Lemma 3 the equalities $\Sigma=\sigma\left(H_{\mathrm{ch}}\right)$ and $\sigma_{\text {two }}=\cup_{p \in \mathbb{T}^{3}} \sigma_{d}(H(p))$ hold.
Remark 6. Note that for any $a, b \in L^{2}\left(\mathbb{T}^{3}\right)$ the operators

$$
\begin{aligned}
& L_{a} R_{22}^{0}(z) L_{b}^{*}\left(L_{s a} R_{22}^{0}(z) L_{s b}^{*}\right) \text { resp., } \\
& L_{a} R_{22}^{0}(z) L_{s b}^{*}\left(L_{s a} R_{22}^{0}(z) L_{b}^{*}\right)
\end{aligned}
$$

is the multiplication operator by the function

$$
\int_{\mathbb{T}^{3}} \frac{a(s) \overline{b(s)} d s}{E(p, s)-z}\left(\int_{\mathbb{T}^{3}} \frac{a(s) \overline{b(s)} d s}{E(s, p)-z}\right),
$$

respectively, is the integral operator with the kernel

$$
\frac{a(s) \overline{b(p)}}{E(p, s)-z}\left(\frac{a(s) \overline{b(q)}}{E(s, p)-z}\right)
$$

in $L^{2}\left(\mathbb{T}^{3}\right)$.

One may check that the operator

$$
\begin{aligned}
D(z)= & I_{1}-L_{\varphi} R_{22}^{0}(z) L_{\varphi}^{*} H_{11}^{0}-z I_{1} \\
& -\frac{1}{2} L_{b} R_{22}^{0}(z) L_{b}^{*}-\frac{1}{2} L_{\varphi} R_{22}^{0}(z) L_{b}^{*}
\end{aligned}
$$

is a multiplication operator by the function $\Delta(\cdot ; z)$ in the space $L^{2}\left(\mathbb{T}^{3}\right)$, where $R_{22}^{0}(z)=\left(H_{22}^{0}-z I_{2}\right)^{-1} ; z \in \mathbb{C} \backslash\left[E_{\min }, E_{\max }\right]$ is the resolvent of $H_{22}^{0}$.

4.1. Faddeev Type Integral Equation. Set $\overline{\mathscr{H}}=\overline{\mathscr{H}}_{0} \oplus \overline{\mathscr{H}}_{1} \oplus \overline{\mathscr{H}}_{2}$, $\overline{\mathscr{H}}_{0}=\mathscr{H}_{0}, \overline{\mathscr{H}}_{1}=\mathscr{H}_{1}$, and $\overline{\mathscr{H}}_{2}=\mathscr{H}_{1}$.

Let the following matrix operators $A(z)$ and $K(z)$, for any $z \in \mathbb{C} \backslash \Sigma$, act in the space $\overline{\mathscr{H}}$ by form

$$
\begin{aligned}
& A(z)=\left(\begin{array}{ccc}
A_{00}(z) & 0 & 0 \\
0 & A_{11}(z) & A_{12}(z) \\
0 & A_{21}(z) & A_{22}(z)
\end{array}\right), \\
& K(z)=\left(\begin{array}{ccc}
K_{00}(z) & K_{01}(z) & 0 \\
K_{10}(z) & K_{11}(z) & K_{12}(z) \\
0 & K_{21}(z) & K_{22}(z)
\end{array}\right)
\end{aligned}
$$

where $A_{i j}(z): \overline{\mathscr{H}}_{j} \rightarrow \overline{\mathscr{H}}_{i}, i, j=0,1,2$, is multiplication by the function $a_{i j}(p, z)$ :

$$
\begin{gathered}
a_{00}(p, z) \equiv 1, \\
a_{11}(p, z)=H_{11}-z I_{1}-\frac{1}{2} L_{b} R_{22}^{0}(z) L_{b}^{*}, \\
a_{12}(p, z)=\frac{1}{\sqrt{2}} L_{b} R_{22}^{0}(z) L_{\varphi}^{*}, \\
a_{21}(p, z)=\overline{a_{12}(p, z)}=\frac{1}{\sqrt{2}} L_{\varphi} R_{22}^{0}(z) L_{b}^{*}, \\
a_{22}(p, z)=I_{1}-L_{\varphi} R_{22}^{0}(z) L_{\varphi}^{*},
\end{gathered}
$$


and the operators $K_{i j}(z): \overline{\mathscr{H}}_{j} \rightarrow \overline{\mathscr{H}}_{i}, i, j=0,1,2$ are defined by

$$
\begin{aligned}
& K_{00}(z)=(z+1) I_{0}-H_{00}, \quad K_{01}(z)=-\mathbf{a}, \\
& K_{10}(z)=K_{01}^{*}(z), \quad K_{11}(z)=-\frac{1}{2} L_{b} R_{22}^{0}(z) L_{s b}^{*}, \\
& K_{12}(z)=\frac{1}{\sqrt{2}} L_{b} R_{22}^{0}(z) L_{s \varphi}^{*}, \quad K_{21}(z)=K_{12}^{*}(z), \\
& K_{22}(z)=-L_{\varphi} R_{22}^{0}(z) L_{s \varphi}^{*} .
\end{aligned}
$$

Note that $K_{i j}(z)$ is Hilbert-Schmidt operators for any $z \in$ $\mathbb{C} \backslash \Sigma$ and so is $K(z)$ in $\overline{\mathscr{H}}$.

Lemma 7. The operator $A(z), z \in \mathbb{C} \backslash \Sigma$, is bounded and invertible, and the inverse operator $A^{-1}(z)$ has form

$$
A^{-1}(z)=\left(\begin{array}{ccc}
B_{00}(z) & 0 & 0 \\
0 & B_{11}(z) & B_{12}(z) \\
0 & B_{21}(z) & B_{22}(z)
\end{array}\right)
$$

where $B_{i j}(z): \overline{\mathscr{H}}_{j} \rightarrow \overline{\mathscr{H}}_{i}, i, j=0,1,2$, is a multiplication operator by the function $b_{i j}(p, z)$ :

$$
\begin{aligned}
& b_{00}(p, z) \equiv 1, \quad b_{11}(p, z)=\frac{a_{22}(p, z)}{\Delta(p ; z)} \\
& b_{12}(p, z)=-\frac{a_{12}(p, z)}{\Delta(p ; z)}, \quad b_{21}(p, z)=-\frac{a_{21}(p, z)}{\Delta(p ; z)}, \\
& b_{22}(p, z)=\frac{a_{11}(p, z)}{\Delta(p ; z)} .
\end{aligned}
$$

Moreover, for any $z \in \mathbb{C} \backslash \Sigma$ the operator $\widehat{T}(z)=A^{-1}(z) K(z)$ is compact.

Proof. Due to the definition of $A(z)$ this operator is multiplication by matrix

$$
A(p, z)=\left(\begin{array}{ccc}
a_{00}(p, z) & 0 & 0 \\
0 & a_{11}(p, z) & a_{12}(p, z) \\
0 & a_{12}(p, z) & a_{22}(p, z)
\end{array}\right) .
$$

Clearly, $A(p, z)$ is continuous matrix-valued function in $\mathbb{T}^{3}$, a fact making the boundedness of $A(z)$.

Since $\operatorname{det}(A(p, z))=\Delta(p ; z)$ and $\Delta(p ; z) \neq 0, p \in \mathbb{T}^{3}$ for $z \notin \Sigma$, then $\operatorname{det}(A(p, z)) \neq 0$.

Consequently, for any $p \in \mathbb{T}^{3}$ the matrix $A(p, z)$ is invertible and

$$
A^{-1}(p, z)=\left(\begin{array}{ccc}
a_{00}(p, z) & 0 & 0 \\
0 & \frac{a_{22}(p, z)}{\Delta(p ; z)} & -\frac{a_{12}(p, z)}{\Delta(p ; z)} \\
0 & -\frac{a_{21}(p, z)}{\Delta(p ; z)} & \frac{a_{11}(p, z)}{\Delta(p ; z)}
\end{array}\right),
$$

which gives a fact that the inverse operator $A^{-1}(z)$ is multiplication by $A^{-1}(p, z)$ in $\overline{\mathscr{H}}$.
Since every component of $K(z)$ is compact so is $K(z)$, a fact that together boundedness of $A^{-1}(z)$ ends the proof.

Next assertion establishes connection between eigenvalues of the operators $H$ and $\widehat{T}(z)=A^{-1}(z) K(z)$.

Lemma 8. The number $z \in \mathbb{C} \backslash \Sigma$ is an eigenvalue of the operator $H$ if and only if the number $\lambda=1$ is an eigenvalue of $\widehat{T}(z)$.

Proof.

Necessity. Let $z \in \mathbb{C} \backslash \Sigma$ be an eigenvalue of $H$ with a corresponding eigenfunction $f$; that is, a system

$$
\begin{aligned}
& \left(H_{00}-z\right) f_{0}+H_{01} f_{1}=0, \\
& H_{10} f_{0}+\left(H_{11}-z\right) f_{1}+H_{12} f_{2}=0, \\
& H_{21} f_{1}+\left(H_{22}-z\right) f_{2}=0,
\end{aligned}
$$

has a nontrivial solution $f=\left(f_{0}, f_{1}, f_{2}\right)$.

Whereas for $z \notin\left[E_{\min }, E_{\max }\right]$ the resolvent $R_{22}^{0}(z)=$ $\left(H_{22}^{0}-z I_{2}\right)$ exists from the third equation of (39) for $f_{2}$, we obtain

$$
f_{2}=R_{22}^{0}(z)\left(\frac{1}{\sqrt{2}}\left(L_{\varphi}^{*}-L_{s \varphi}^{*}\right) c-\frac{1}{2}\left(L_{b}^{*}-L_{s b}^{*}\right) f_{1}\right),
$$

where

$$
c=\sqrt{2} L_{\varphi} f_{2}
$$

since $L_{s \varphi} f_{2}(q)=-L_{s \varphi} f_{2}(q)$.

Now we change (39) to the equivalent system of equations, using $f_{0}, f_{1}$, and $c$. For this purpose, putting (40) for $f_{2}$ in (39), (41), we get

$$
f_{0}=\left((z+1) I_{0}-H_{00}\right) f_{0}-\mathbf{a} f_{1},
$$

$$
\begin{gathered}
\left(H_{11}-z I_{1}-\frac{1}{2} L_{b} R_{22}^{0}(z) L_{b}^{*}\right) f_{1}+\frac{1}{\sqrt{2}} L_{b} R_{22}^{0}(z) L_{\varphi}^{*} c \\
=-\mathbf{a}^{*} f_{0}-\frac{1}{2} L_{b} R_{22}^{0}(z) L_{s b}^{*} f_{1}+\frac{1}{\sqrt{2}} L_{b} R_{22}^{0}(z) L_{s \varphi}^{*} c, \\
\frac{1}{\sqrt{2}} L_{\varphi} R_{22}^{0}(z) L_{b}^{*} f_{1}+\left(I_{1}-L_{\varphi} R_{22}^{0}(z) L_{\varphi}^{*}\right) c \\
=\frac{1}{\sqrt{2}} L_{\varphi} R_{22}^{0}(z) L_{s b}^{*} f_{1}-L_{\varphi} R_{22}^{0}(z) L_{s \varphi}^{*} c ;
\end{gathered}
$$

that is, due to (32), (33), and (34)

$$
A(z) \psi=K(z) \psi, \quad \psi=\left(f_{0}, f_{1}, c\right) \in \overline{\mathscr{H}} .
$$

The last fact and Lemma 7 conclude

$$
\psi=A^{-1}(z) K(z) \psi, \quad \text { i.e., } \psi=\widehat{T}(z) \psi \text {. }
$$

The system (39) has nontrivial solution, so is the system (44), a fact implying that the number 1 is an eigenvalue of the operator $\widehat{T}(z)$.

Sufficiency. Let $\widehat{T}(z), z \in \mathbb{C} \backslash \Sigma$, have an eigenvalue $\lambda=1$ with corresponding eigenfunction $\psi=\left(f_{0}, f_{1}, c\right) \in \overline{\mathscr{H}}$. Thus (44) 
has nontrivial solution. Multiplying (44) with the operator $A(z)$ from left side we get $A(z) \psi=K(x) \psi$; that is,

$$
\begin{aligned}
& \quad\left(H_{00}-z I_{0}\right) f_{0}+\mathbf{a} f_{1}=0, \\
& \mathbf{a}^{*} f_{0}+\left(H_{11}-z I_{1}\right) f_{1}+W f_{1} \\
& \quad+L_{b}\left(R_{22}^{0}(z)\left(\frac{1}{\sqrt{2}}\left(L_{\varphi}^{*}-L_{\varphi}^{*}\right) c-\frac{1}{2}\left(L_{b}^{*}-L_{s b}^{*}\right) f_{1}\right)\right) \\
& =0, \\
& c=L_{\varphi}\left(R_{22}^{0}(z)\left(\frac{1}{\sqrt{2}}\left(L_{\varphi}^{*}-L_{\varphi}^{*}\right) c-\frac{1}{2}\left(L_{b}^{*}-L_{s b}^{*}\right) f_{1}\right)\right) .
\end{aligned}
$$

Set

$$
f_{2}=R_{22}^{0}(z)\left(\frac{1}{\sqrt{2}}\left(L_{\varphi}^{*}-L_{s \varphi}^{*}\right) c-\frac{1}{2}\left(L_{b}^{*}-L_{s b}^{*}\right) f_{1}\right) .
$$

Then the last two equations of (45) give

$$
c=\sqrt{2} L_{\varphi} f_{2} .
$$

It is easy to see that the vector function $f=\left(f_{0}, f_{1}, f_{2}\right) \in$ $\mathscr{H}$ satisfies the equation $H f=z f$; that is, the number $z$ is an eigenvalue of $H$.

\section{Formulation and Proof of the Main Results}

The first main result of the paper is given in the following theorem.

Theorem 9. The essential spectrum $\sigma_{\text {ess }}(H)$ of $H$ coincides with the set $\Sigma$; that is,

$$
\sigma_{\text {ess }}(H)=\Sigma \text {. }
$$

Proof.

First Part. $\sigma_{\text {two }} \subset \sigma_{\text {ess }}(H)$. This inclusion will be showed using the Weyl criterion (see [22, VII.12]) by constructing an appropriate sequence of trial functions.

Let $z_{0} \in \Sigma$. First we consider case $z_{0} \in \sigma_{\text {two }}$ and $z_{0} \notin$ $\left[E_{\min }, E_{\max }\right]$.

Then due to Theorem 2 and Lemma 3 there exists $p_{0} \in \mathbb{T}^{3}$ such that $\operatorname{det}\left(A\left(p_{0}, z_{0}\right)\right)=\Delta\left(p_{0}, z_{0}\right)=0$ and the system of equations,

$$
\begin{gathered}
\alpha_{0}=0 \\
\left(H_{00}-z_{0} I_{0}-\frac{1}{2} \mathbf{b} r_{0}\left(p_{0}, z_{0}\right) \mathbf{b}^{*}\right) \alpha_{1} \\
+\frac{1}{\sqrt{2}} \mathbf{b} r_{0}\left(p_{0}, z_{0}\right) \boldsymbol{\varphi}^{*} \alpha_{2}=0 \\
\frac{1}{\sqrt{2}} \boldsymbol{\varphi} r_{0}\left(p_{0}, z_{0}\right) \mathbf{b}^{*} \alpha_{1}+\left(I_{0}-\boldsymbol{\varphi} r_{0}\left(p_{0}, z_{0}\right) \boldsymbol{\varphi}^{*}\right) \alpha_{2}=0
\end{gathered}
$$

has an infinite number solutions, and some solutions have form $\mathbf{l}=\left(0, \alpha_{1}, \alpha_{2}\right)$ with the condition $\alpha_{1}^{2}+\alpha_{2}^{2}>0$.
Then from (49) we obtain

$$
A\left(p_{0}, z_{0}\right) \mathbf{l}=0, \quad \mathbf{l}=\left(0, \alpha_{1}, \alpha_{2}\right) \in \mathbb{C}^{3}
$$

Let $\chi_{n}(p), n \in \mathbb{N}$, be a characteristic function of a set

$$
V_{n}=\left\{p \in \mathbb{T}^{3}: \frac{1}{n+1}<\left|p-p_{0}\right|<\frac{1}{n}\right\},
$$

and $\mu\left(V_{n}\right)$ Lebesgue measure of $V_{n}$.

A sequences of trial functions $\left\{f^{(n)}\right\}$ is chosen by

$$
f^{(n)}=\left(0, f_{1}^{(n)}, f_{2}^{(n)}\right),
$$

where

$$
\begin{aligned}
& f_{1}^{(n)}=\alpha_{1} \psi^{(n)}, \\
& f_{2}^{(n)}=R_{22}^{0}\left(z_{0}\right)\left(\alpha_{2}\left(L_{\varphi}^{*}-L_{s \varphi}^{*}\right) \psi^{(n)}-\frac{\alpha_{1}}{2}\left(L_{b}^{*}-L_{s b}^{*}\right) \psi^{(n)}\right), \\
& \psi^{(n)}=\frac{c_{n} \chi_{n}}{\sqrt{\mu\left(V_{n}\right)}} .
\end{aligned}
$$

Here the function $c_{n} \in L^{2}\left(\mathbb{T}^{3}\right)$ is found from the orthogonality condition $\left\{f^{(n)}\right\}$; that is,

$$
\begin{aligned}
& \left\langle f^{(n)}, f^{(m)}\right\rangle_{\mathscr{H}^{(3)}} \\
& =-\left\langle R_{22}^{0}\left(z_{0}\right)\left(\alpha_{2} L_{\varphi}^{*}-\frac{\alpha_{1}}{2} L_{b}^{*}\right) \psi^{(n)},\right. \\
& \left.R_{22}^{0}\left(z_{0}\right)\left(\alpha_{2} L_{s \varphi}^{*}-\frac{\alpha_{1}}{2} L_{s b}^{*}\right) \psi^{(m)}\right\rangle_{2} \\
& =0, \quad n \neq m,
\end{aligned}
$$

since

$$
\begin{aligned}
\left\langle R_{22}^{0}\left(z_{0}\right) L_{\alpha}^{*} \psi^{(n)}, R_{22}^{0}\left(z_{0}\right) L_{\beta}^{*} \psi^{(m)}\right\rangle_{2} & =0, \\
\left\langle R_{22}^{0}\left(z_{0}\right) L_{s \alpha}^{*} \psi^{(n)}, R_{22}^{0}\left(z_{0}\right) L_{s \beta}^{*} \psi^{(m)}\right\rangle_{2} & =0, \\
n & \neq m,
\end{aligned}
$$

where the notation $\alpha, \beta$ admits function-value either $b$ or $\varphi$.

There existence of $c_{n}$ follows from the following assertion, and they may be constructed by the method of induction.

Proposition 10. There exists orthonormal system $\left\{c_{n}\right\} \subset$ $L^{2}\left(\mathbb{T}^{3}\right)$, satisfying conditions

(a) supp $c_{n} \subset V_{n}$,

(b) $\left\langle R_{22}^{0}\left(z_{0}\right)\left(\alpha_{2} L_{\varphi}^{*}-\frac{\alpha_{1}}{2} L_{b}^{*}\right) \psi^{(n)}\right.$,

$$
\left.R_{22}^{0}\left(z_{0}\right)\left(\alpha_{2} L_{s \varphi}^{*}-\frac{\alpha_{1}}{2} L_{s b}^{*}\right) \psi^{(m)}\right\rangle_{2}=0 .
$$


Proof. Let $c_{1}=\chi_{1} / \sqrt{\mu\left(V_{1}\right)}$. Then we chose $\widetilde{c}_{2} \in L^{2}\left(V_{2}\right)$ so that $\left\|\widetilde{\mathcal{c}}_{2}\right\|=1\left(\widetilde{\mathcal{c}}_{2}, \varepsilon_{1}^{(2)}\right)=0$, where

$\varepsilon_{1}^{(2)}(p)$

$$
\begin{aligned}
=\int_{\mathbb{T}^{3}} & \left(\frac{\left[\alpha_{2} \varphi(q)-\left(\alpha_{1} / 2\right) b(q)\right]\left[\left(\alpha_{1} / 2\right) b(p)-\alpha_{2} \varphi(p)\right]}{\left(E(p, q)-z_{0}\right)^{2}}\right) \\
& \times c_{1}(q) \chi_{2}(q) d q .
\end{aligned}
$$

Set $c_{2}(p)=\widetilde{c}_{2}(p) \chi_{2}(p)$. We continue this process. Assume that we have constructed $c_{1}(p), \ldots, c_{n}(p)$. Then a function $\widetilde{c}_{n+1} \in L^{2}\left(V_{n+1}\right)$ is chosen so that $\left\|\widetilde{c}_{n+1}\right\|=1$ and it is orthogonal to

$$
\begin{aligned}
& \varepsilon_{i}^{(n+1)}(p) \\
& =\int_{\mathbb{T}^{3}}\left(\frac{\left[\alpha_{2} \varphi(q)-(1 / 2) \alpha_{1} b(q)\right]\left[\alpha_{1} b(p)-2 \alpha_{2} \varphi(p)\right]}{\left(E(p, q)-z_{0}\right)^{2}}\right) \\
& \quad \times c_{i}(q) \chi_{n+1}(q) d q, \quad i=\overline{1, n} .
\end{aligned}
$$

$\operatorname{Set}_{n+1}(p)=\widetilde{c}_{n+1}(p) \chi_{n+1}(p)$. Thus we have built orthonormal system $\left\{c_{n}\right\}$, satisfying the conditions of the proposal.

Continuation of the Proof of Theorem 9. Assume $\Delta\left(p_{0}, z_{0}\right)=0$. Then

$$
\left\|f^{(n)}\right\|^{2} \geq \frac{\xi_{0}}{2 \mu\left(V_{n}\right)}, \quad \xi_{0}=\alpha_{1}^{2}+\frac{\alpha_{2}^{2}\left\|\varphi_{2}\right\|^{2}}{\left(E_{\max }-z_{0}\right)^{2}} .
$$

Define an orthonormal system $\widetilde{f}^{(n)}=f^{(n)} /\left\|f^{(n)}\right\|$.

We estimate the norm of $\left(H-z_{0}\right) \tilde{f}^{(n)}$ as

$$
\begin{aligned}
\left\|\left(H-z_{0}\right) \widetilde{f}^{(n)}\right\|^{2} & \leq\left\|A\left(z_{0}\right) \widetilde{\psi}^{(n)}\right\|_{\overline{\mathscr{C}}}^{2}+\left\|K\left(z_{0}\right) \widetilde{\psi}^{(n)}\right\|_{\mathscr{\mathscr { H }}^{2}}^{2}, \\
\widetilde{\psi}^{(n)} & =\left(0, \frac{\psi_{1}^{(n)}}{\left\|f^{(n)}\right\|}, \frac{\psi_{2}^{(n)}}{\left\|f^{(n)}\right\|}\right) .
\end{aligned}
$$

Note that $\left\{\widetilde{\psi}^{(n)}\right\} \subset \overline{\mathscr{H}}$ is an orthogonal system, a fact that follows from disjointness of the supports of $\widetilde{\psi}^{(n)}$ and $\widetilde{\psi}^{(m)}$.

From the equality,

$$
\left\|\widetilde{\psi}^{(n)}\right\|^{2}=\frac{1}{\left\|f^{(n)}\right\|^{2}} \frac{1}{\mu\left(V_{n}\right)}\left(\alpha_{1}^{2}+\alpha_{2}^{2}\right),
$$

and (59) we take the uniformly boundedness of the system $\left\{\widetilde{\psi}^{(n)}\right\}$; that is, $\left\|\widetilde{\psi}^{(n)}\right\|^{2} \leq\left(1 / \xi_{0}\right)\|\mathbf{1}\|_{\mathbb{C}^{3}}^{2}$ for all $n \in \mathbb{N}$.

Then, due to the compactness of $K\left(z_{0}\right)$, the limit $\left\|K\left(z_{0}\right) \widetilde{\psi}^{(n)}\right\| \rightarrow 0$, as $n \rightarrow \infty$, holds.

Now we estimate $\left\|A\left(z_{0}\right) \widetilde{\psi}^{(n)}\right\|$. Using the Schwartz inequality we receive

$$
\begin{aligned}
& \left\|A\left(z_{0}\right) \widetilde{\psi}^{(n)}\right\|^{2} \leq C^{2} \sup _{p \in V_{n}}\left\|A\left(p, z_{0}\right) 1\right\|_{\mathbb{C}^{3}}^{2}, \\
& \text { where } C^{2}=\max \left\{\frac{2}{\xi_{0}}, \frac{8\|\varphi\|^{2}}{\xi_{0}}\right\} .
\end{aligned}
$$

By the continuity of the matrix-valued function $F(p)=$ $A\left(p, z_{0}\right)$, the limit $\sup _{p \in V_{n}}\left\|A\left(p, z_{0}\right) \mathbf{l}\right\|_{\mathbb{C}^{3}} \rightarrow 0$, as $n \rightarrow \infty$, is obtained.

Therefore, the sequence of orthonormal vector functions $\left\{\tilde{f}^{(n)}\right\}$ satisfies $\left\|\left(H-z_{0}\right) \widetilde{f}^{(n)}\right\| \rightarrow 0$, as $n \rightarrow \infty$, a fact that means $z_{0} \in \sigma_{\text {ess }}(H)$. Consequently, $\sigma_{\text {two }} \subset \sigma_{\text {ess }}(H)$ since $z_{0}$ is arbitrary.

In case $z_{0} \in\left[E_{\min }, E_{\max }\right]$, we chose $\left(p_{0}, q_{0}\right) \in\left(\mathbb{T}^{3}\right)^{2}$ as $z=E\left(p_{0}, q_{0}\right)$ and we can construct an appropriate sequence of trial functions as

$$
\begin{gathered}
f^{(n)}=\left(0,0, f_{2}^{(n)}\right)^{T}, \\
f_{2}^{(n)}(p, q)=\operatorname{sgn}\left(p^{2}-q^{2}\right) \frac{\chi_{n}(p, q)}{\mu\left(U_{n}\right)},
\end{gathered}
$$

where $\chi_{n}(p, q)$ is a characteristic function of the set $U_{n}=V_{n} \times$ $V_{n} \subset\left(\mathbb{T}^{3}\right)^{2}, \mu\left(U_{n}\right)$ is Lebesgue measure of $U_{n}$, and sgn is sign function.

Consequently, $\left[E_{\min }, E_{\max }\right] \cup \sigma_{\text {two }} \subset \sigma_{\text {ess }}(H)$.

Second Part. $\sigma_{\text {ess }}(H) \subset \Sigma$. Denote by

$$
R(z)=\left(\begin{array}{lll}
R_{00}(z) & R_{01}(z) & R_{02}(z) \\
R_{10}(z) & R_{11}(z) & R_{12}(z) \\
R_{20}(z) & R_{21}(z) & R_{22}(z)
\end{array}\right), \quad z \in \Sigma,
$$

the resolvent form of $H$, where $R_{i j}(z): \mathscr{H}_{j} \rightarrow \mathscr{H}_{i}, i \cdot j=$ $1,2,3$, is its matrix "entries," it's rows $R_{i}(z): \mathscr{H}^{(3)} \rightarrow \mathscr{H}_{i}, i=$ $1,2,3$ we denote by

$$
R_{i}(z)=\left(R_{i 0}(z), R_{i 1}(z), R_{i 2}(z)\right) .
$$

\section{Setting}

$$
\begin{aligned}
& \mathscr{R}_{0}(z)=\operatorname{diag}\left\{I_{0}, I_{1}, R_{22}^{0}(z)\right\}, \\
& H_{0}(z)=\operatorname{diag}\left\{I_{0}, I_{1}, H_{22}^{0}-z I_{2}\right\},
\end{aligned}
$$

where $R_{22}^{0}(z)=\left(H_{22}^{0}-z I_{2}\right)^{-1}, z \in \mathbb{C} \backslash\left[E_{\min }, E_{\max }\right]$, is the resolvent of the operator $H_{22}^{0}$, we obtain the resolvent equation

$$
R(z)=\mathscr{R}_{0}(z)-\mathscr{R}_{0}(z)\left((H-z I)-H_{0}(z)\right) R(z) .
$$

In our case resolvent Equation (67) is not compact. Therefore to overcome this difficulty, we derive Faddeev type system of integral equations (see, e.g., [23]) for the components of the resolvent. 
We write (67) as the form

$$
\begin{aligned}
& \left\{\begin{array}{l}
R_{0}(z) \\
R_{1}(z) \\
R_{2}(z)
\end{array}\right) \\
& =\mathscr{R}_{0}(z)-\mathscr{R}_{0}(z) \\
& \times\left(\begin{array}{ccc}
H_{00}-(z+1) I_{0} & H_{01} & 0 \\
H_{10} & H_{11}-(z+1) I_{1} & H_{12} \\
0 & H_{21} & \left(H_{22}-H_{22}^{0}\right)
\end{array}\right) \\
& \times\left(\begin{array}{l}
R_{0}(z) \\
R_{1}(z) \\
R_{2}(z)
\end{array}\right) ;
\end{aligned}
$$

that is,

$$
\begin{aligned}
& \left(\begin{array}{l}
R_{0}(z) \\
R_{1}(z) \\
R_{2}(z)
\end{array}\right) \\
= & R_{0}(z) \\
& -\left(\begin{array}{c}
H_{10} R_{0}(z)+\left(H_{11}-z I_{1}-I_{1}\right) R_{1}(z)+H_{12} R_{2}(z) \\
R_{22}^{0}(z) H_{21} R_{1}(z)-R_{22}^{0}(z)\left(L_{\varphi}^{*} L_{\varphi}+L_{s \varphi}^{*} L_{s \varphi}\right) R_{2}(z)
\end{array}\right) .
\end{aligned}
$$

Defining the unitary operator $P: L_{\text {as }}^{2}\left(\left(\mathbb{T}^{3}\right)^{2}\right) \rightarrow$ $L_{\mathrm{as}}^{2}\left(\left(\mathbb{T}^{3}\right)^{2}\right): P f(p, q)=-f(q, p)$, we can take $P L_{s \varphi}^{*} L_{s \varphi}=$ $-L_{\varphi}^{*} L_{\varphi}$ and an equality

$$
L_{s \varphi}^{*} L_{s \varphi} R_{2}(z)=-L_{s \varphi}^{*} P L_{\varphi} R_{2}(z)
$$

Then, putting the third equation

$$
\begin{aligned}
R_{2}(z)=R_{22}^{0}(z)- & \left(R_{22}^{0}(z) H_{21} R_{1}(z)\right. \\
& \left.-R_{22}^{0}(z)\left(L_{\varphi}^{*}-L_{s \varphi}^{*} P\right) L_{\varphi} R_{2}(z)\right),
\end{aligned}
$$

of (69) into $H_{12} R_{2}(z)$, we take

$$
R(z)=\mathscr{R}_{0}(z)-Q_{0}(z)-H_{1} \mathbf{X}(z)
$$

where

$$
\begin{aligned}
Q_{0}(z) & =\operatorname{diag}\left\{0, H_{12} R_{22}^{0}(z), 0\right\}, \\
\mathbf{X}(z) & =\left(R_{0}(z), R_{1}(z), L_{\varphi} R_{2}(z)\right)^{T},
\end{aligned}
$$

is an unknown vector operator and $H_{1}: \overline{\mathscr{H}} \rightarrow \overline{\mathscr{H}}$ has the form

$$
H_{1}=\left(\begin{array}{ccc}
H_{00}-(z+1) I_{0} & H_{01} & 0 \\
H_{10} & H_{11}-(z+1) I_{1}-H_{12} R_{22}^{0}(z) H_{21} & H_{12} R_{22}^{0}(z)\left(L_{\varphi}^{*}-L_{s \varphi}^{*} P\right) \\
0 & R_{22}^{0}(z) H_{21} & -R_{22}^{0}(z)\left(L_{\varphi}^{*}-L_{s \varphi}^{*} P\right)
\end{array}\right)
$$

Set

$$
L(z)=\operatorname{diag}\left\{I_{0}, I_{1}, L_{\varphi}\right\}
$$
as

$$
\begin{aligned}
\mathbf{X}(z)= & L(z) \mathscr{R}_{0}(z)-L(z) Q_{0}(z) \\
& -\left(\begin{array}{ccc}
H_{00}-(z+1) I_{0} & H_{01} & 0 \\
H_{10} & H_{11}-(z+1) I_{1}-H_{12} R_{22}^{0}(z) H_{21} & H_{12} R_{22}^{0}(z)\left(L_{\varphi}^{*}-L_{s \varphi}^{*} P\right) \\
0 & L_{\varphi} R_{22}^{0}(z) H_{21} & -L_{\varphi} R_{22}^{0}(z)\left(L_{\varphi}^{*}-L_{s \varphi}^{*} P\right)
\end{array}\right) \mathbf{X}(z) .
\end{aligned}
$$


Due to (33), (34) we have

$$
\begin{gathered}
H_{00}-(z+1) I_{0}=-I_{0}-A_{00}(z)-B_{00}(z), \\
H_{11}-(z+1) I_{1} \\
-H_{12} R_{22}^{0}(z) H_{21}=-I_{1}-A_{11}(z)-K_{11}(z), \\
-H_{12} R_{22}^{0}(z)\left(L_{\varphi}^{*}-L_{s \varphi}^{*} P\right)=A_{12}(z)-K_{12}(z) \\
L_{\varphi} R_{22}^{0}(z) H_{21}=A_{21}(z)-K_{21}(z), \\
L_{\varphi} R_{22}^{0}(z)\left(L_{\varphi}^{*}-L_{s \varphi}^{*} P\right)=-I_{2}+A_{22}(z)-K_{22}(z) .
\end{gathered}
$$

Using these and (33), (34) we get

$$
(A(z)-K(z)) \mathbf{X}(z)=L(z)\left(\mathscr{R}_{0}(z)-Q_{0}(z)\right) .
$$

By virtue of Lemma 7 the operator $A(z), z \in \mathbb{C} \backslash \Sigma$, is invertible and then we get the Faddeev type equation [23]

$$
\left(I-A^{-1}(z) K(z)\right) \mathbf{X}(z)=A^{-1}(z) L(z)\left(\mathscr{R}_{0}(z)-Q_{0}(z)\right),
$$

where $I$ is the identity operator on $\overline{\mathscr{H}}$.

The operator $\widehat{T}(z)=A^{-1}(z) K(z)$ is a compact operatorvalued function on $\mathbb{C} \backslash \Sigma$ and $F(z)=I-\widehat{T}(z)$ is invertible; even $z$ is real and either very negative or very positive. The analytic Fredholm theorem (see, e.g., in [22, Theorem VI.14]) implies that there is a discrete set $S \subset \mathbb{C} \backslash \Sigma$ such that $F(z)^{-1}$ exists and is analytic in $\mathbb{C} \backslash(\Sigma \cup S)$ and meromorphic in $\mathbb{C} \backslash \Sigma$ with finite rank residues. Thus the function (I $\widehat{T}(z))^{-1} A^{-1}(z) L(z)\left(\mathscr{R}_{0}(z)-Q_{0}(z)\right) \equiv G(z)$ is analytic in $\mathbb{C} \backslash(\Sigma \cup S)$ with finite rank residues at the points of $S$.

Let $z \notin S$ and $\operatorname{Im} z \neq 0$; then, by (81) and (79) we have $G(z)=\mathbf{X}(z)$. In particular,

$$
\begin{aligned}
& R(z)(H-z I) \\
& \quad=\left(\mathscr{R}_{0}(z)-Q_{0}(z)-H_{1} \mathbf{X}(z)\right)(H-z \mathbf{I})=I,
\end{aligned}
$$

where $I$ is the identity operator in $\mathscr{H}^{(3)}$.

By analytic continuation, this holds for any $z \notin \Sigma \cup S$. Thus, for any such $z$, the operator $H-z I$ has a bounded inverse. Therefore $\sigma(H) \backslash \Sigma$ consists of isolated points and only the frontier points of $\Sigma$ are possible their limit points. Finally, since $R(z)$ has finite rank residues at $z \in S$, we conclude that $\sigma(H) \backslash \Sigma$ belongs to the discrete spectrum $\sigma_{d}(H)$ of $H$, which completes the proof of Theorem 9.

Now we derive resolvent form (64) of the operator $H$.

Theorem 11. Let $z \in \mathbb{C} \backslash \sigma(H)$. Then the resolvent $R(z)=$ $(H-z I)^{-1}$ of $H$ has the form

$$
\begin{aligned}
R(z)= & \mathscr{R}_{0}(z)-Q_{0}(z) \\
& -H_{1}(A(z)-K(z))^{-1} L(z)\left(\mathscr{R}_{0}(z)-Q_{0}(z)\right),
\end{aligned}
$$

where $A(z), K(z), \mathscr{R}_{0}(z), H_{1}$, and $L(z)$ are defined by (33), (34), (66), (74), and (75), respectively.

Proof. Lemma 8, (69), and (79) give the proof.

\section{Conflict of Interests}

The authors declare that there is no conflict of interests regarding the publication of this paper.

\section{Acknowledgment}

This work was partially supported by University Putra Malaysia under research university grant scheme (FRGS), and Project code is 5524430. The authors are indebted to the anonymous referees for a number of constructive comments.

\section{References}

[1] R. A. Minlos and H. Spohn, "The three-body problem in radioactive decay: the case of one atom and at most two photons," in TopicS in StatiStical and Theoretical PhySicS. Berezin'S Memory Volume, American Mathematical Society Translation, vol.177, no. 2, pp. 159193, 1996.

[2] V. A. Malyshev and R. A. Minlos, Linear Infinite-Particle Operators, vol. 143, American Mathematical Society, Providence, RI, USA, 1995.

[3] D. C. Mattis, “The few-body problem on a lattice," Reviews of Modern Physics, vol. 58, no. 2, pp. 361-379, 1986.

[4] A. I. Mogilner, "Hamiltonians in solid-state physics as multiparticle discrete Schrödinger operators: problems and results," in Advances in Soviet Mathematics, vol. 5, pp. 139-194, 1991.

[5] K. O. Friedrichs, Perturbation of Spectra in Hilbert Space, American Mathematical Society, Providence, RI, USA, 1965.

[6] C. H. Buhler, S. Yunoki, and A. Moreo, "Magnetic domains and stripes in a spin-fermion model for cuprates," Physical Review Letters, vol. 84, no. 12, Article ID 269026, 2000.

[7] I. M. Sigal, A. Soffer, and L. Zielinski, "On the spectral properties of Hamiltonians without conservation of the particle number," Journal of Mathematical Physics, vol. 43, no. 4, pp. 1844-1855, 2002.

[8] Y. V. Zhukov and R. A. Minlos, "Spectrum and scattering in a "spin-boson" model with not more than three photons," Theoretical and Mathematical Physics, vol. 103, no. 1, pp. 398411, 1995.

[9] S. Albeverio, S. N. Lakaev, and T. H. Rasulov, "On the spectrum of an hamiltonian in fock space. Discrete spectrum asymptotics," Journal of Statistical Physics, vol. 127, no. 2, pp. 191-220, 2007.

[10] S. Albeverio, S. N. Lakaev, and T. H. Rasulov, "The Efimov effect for a model operator associated with the Hamiltonian of a non conserved number of particles," Methods of Functional Analysis and Topology, vol. 13, no. 1, pp. 1-16, 2007.

[11] S. N. Lakaev and T. K. Rasulov, "A model in the theory of perturbations of the essential spectrum of multiparticle operators," Mathematical Notes, vol. 73, no. 3-4, pp. 521-528, 2003.

[12] T. H. Rasulov, "The Faddeev equation and the location of the essential spectrum of a model operator for several particles," Mathematics (Izvestiya VUZ. Matematika), vol. 12, pp. 59-69, 2008.

[13] T. H. Rasulov, "On the structure of the essential spectrum of a model many-body Hamiltonian," Mathematical Notes, vol. 83, no. 1, pp. 86-94, 2008. 
[14] T. H. Rasulov, "Investigation of the spectrum of a model operator in a Fock space," Teoreticheskaya i Matematicheskaya Fizika, vol. 161, no. 2, pp. 164-175, 2009 (Russian).

[15] T. H. Rasulov, "Study of the essential spectrum of a matrix operator," Teoreticheskaya i Matematicheskaya Fizika, vol. 164, no. 1, pp. 62-77, 2010 (Russian).

[16] G. R. Yodgarov and M. I. Muminov, "On the spectrum of a model operator in the theory of perturbations of the essential spectrum," Teoreticheskaya i Matematicheskaya Fizika, vol. 144, no. 3, pp. 544-554, 2005 (Russian).

[17] S.N. Lakaev and M.K. Shermatov, "The spectrum of the Hamiltonian of a system of three quantum particles on a lattice," Uspekhi Matematicheskikh Nauk, vol. 54, no. 6 (330), pp. 165166, 1999.

[18] Z. I. Muminov, F. Ismail, Z. K. Eshkuvatov, and J. Rasulov, "On the discrete spectrum of a model operator in fermionic Fock space," Abstract and Applied Analysis, vol. 2013, Article ID 875194, 12 pages, 2013.

[19] Z. I. Muminov, F. Ismail, and Z. K. Eshkuvatov, "On the number of eigenvalues of a model operator in fermionic Fock space," Journal of Physics: Conference Series, vol. 435, Article ID 012036, 2013.

[20] S. Albeverio, S. N. Lakaev, and Z. I. Muminov, "Schrödinger operators on lattices. The Efimov effect and discrete spectrum asymptotics," Annales Henri Poincaré, vol. 5, no. 4, pp. 743-772, 2004.

[21] M. Reed and B. Simon, Methods of Modern Mathematical Physics, vol. 4 of Analysis of Operators, Academic Press, New York, NY, USA, 1978.

[22] M. Reed and B. Simon, Methods of Modern Mathematical Physics, vol. 1 of Functional Analysis, Academic Press, New York, NY, USA, 1972.

[23] L. D. Faddeev and S. P. Merkuriev, Quantum Scattering Theory for Several Particle Systems, Kluwer Academic, 1993. 


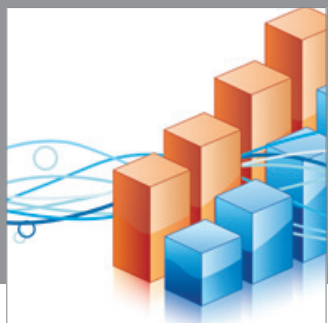

Advances in

Operations Research

mansans

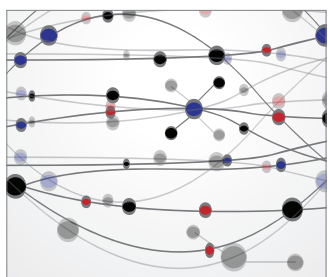

The Scientific World Journal
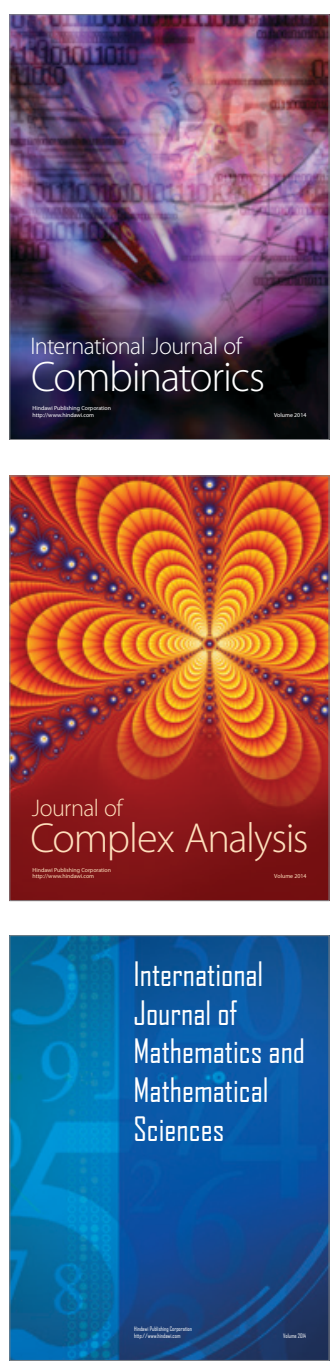
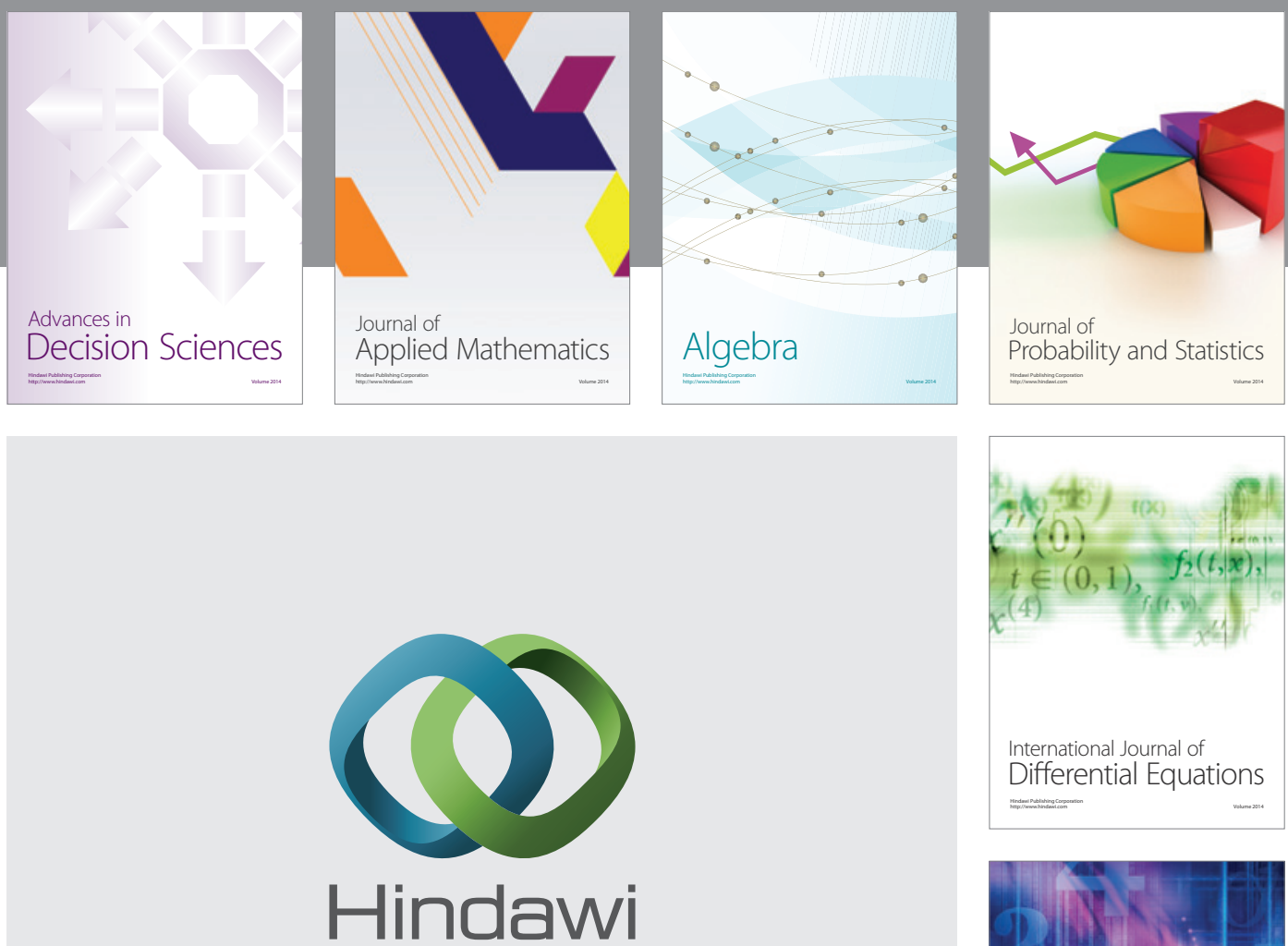

Submit your manuscripts at http://www.hindawi.com
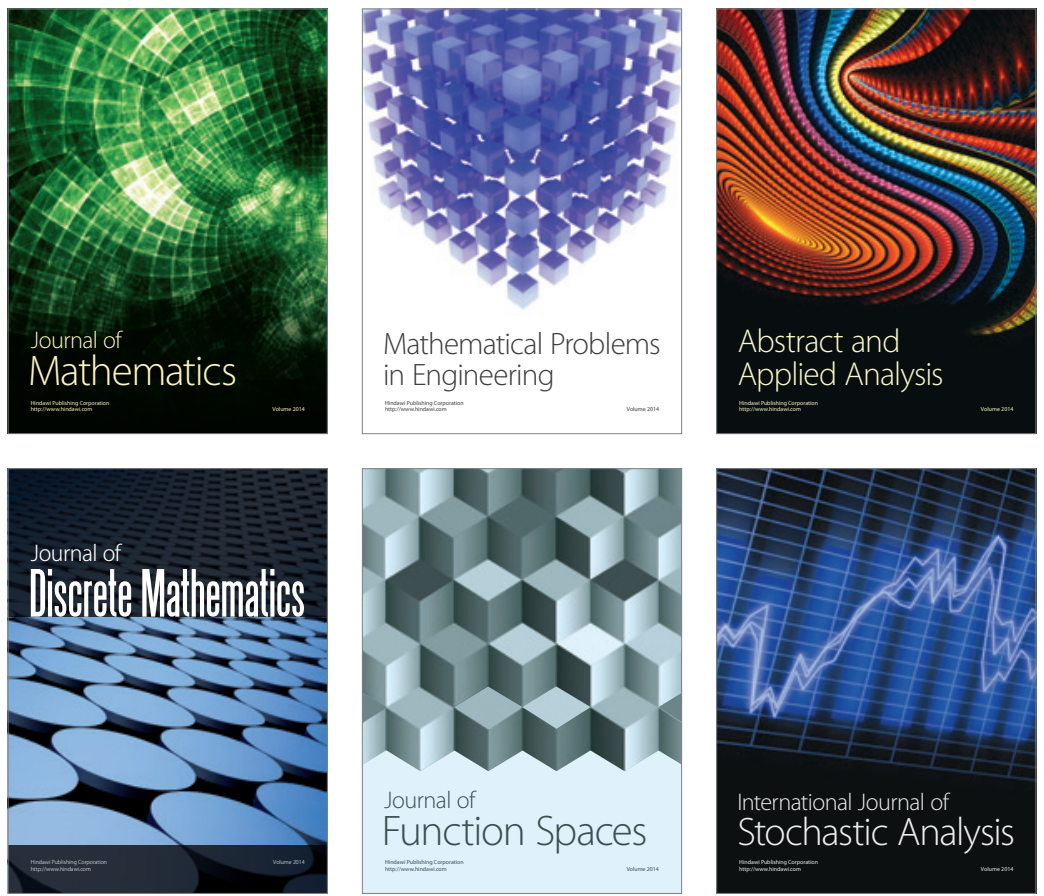

Journal of

Function Spaces

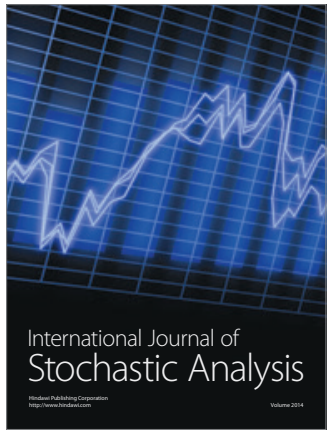

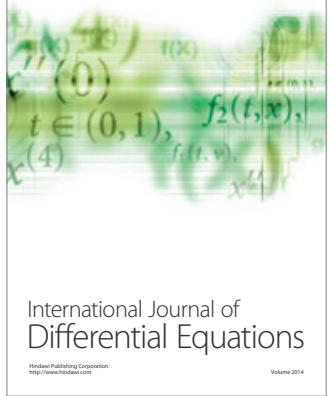
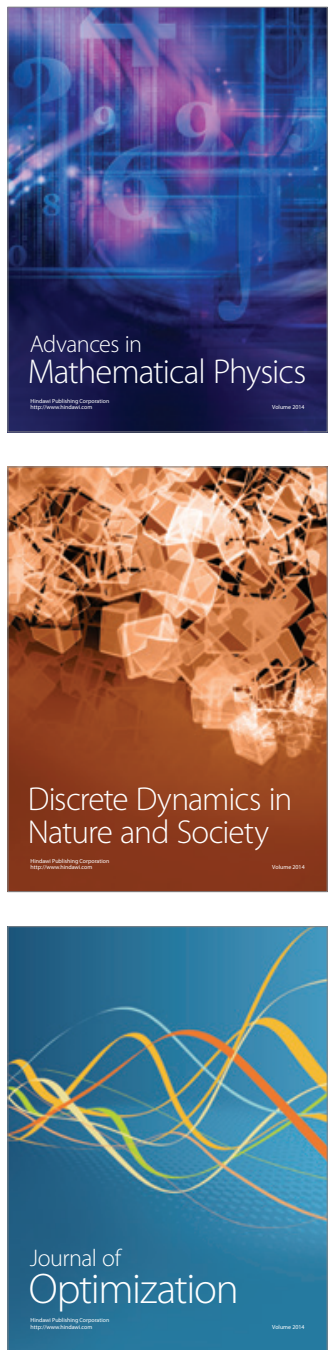